\title{
STURM-LIOUVILLE DIFFERENTIAL OPERATORS WITH DEVIATING ARGUMENT
}

\author{
S. A. BUTERIN, M. PIKULA AND V. A. YURKO
}

\begin{abstract}
Non-selfadjoint second-order differential operators with a constant delay are studied. We establish properties of the spectral characteristics and investigate the inverse problem of recovering operators from their spectra. For this inverse problem the uniqueness theorem is proved.
\end{abstract}

\section{Introduction}

We study an inverse spectral problem for non-selfadjoint Sturm-Liouville differential operators on a finite interval with a constant delay and with complex-valued potentials. Inverse spectral problems consist in recovering operators from their spectral characteristics. The greatest success in the inverse spectral theory has been achieved for the classical SturmLiouville operator (see the monographs [1-5] and the references therein) and afterwards for higher-order differential operators and other classes of differential operators and systems [4]-[7]. The classical methods of inverse spectral theory (transformation operator method [1]-[4] and method of spectral mappings [3]-[6]), which allow obtaining global solutions of inverse problems for differential operators, are not applicable for differential operators with deviating argument as well as for other classes of nonlocal operators such as integrodifferential, integral and other operators. Therefore, the general inverse spectral theory for nonlocal operators has not yet been constructed and there are only isolated results in this direction not forming the general picture [8]-[21].

In the present paper we consider the boundary value problems $L_{j}=L_{j}(q, h), j=0,1$, of the form

$$
\begin{aligned}
-y^{\prime \prime}(x)+q(x) y(x-a) & =\lambda y(x), \quad x \in(0, \pi), \\
y^{\prime}(0)-h y(0) & =y^{(j)}(\pi)=0,
\end{aligned}
$$

Received July 21, 2016, accepted September 2, 2016. 2010 Mathematics Subject Classification. 34A55 34K29 47E05.

Key words and phrases. Differential operators, deviating argument, spectral properties, inverse spectral problems.

Corresponding author: S. A. Buterin. 
where $\lambda$ is the spectral parameter, $a \in(0, \pi), h$ is a complex number, $q(x)$ is a complex-valued function, $q(x) \in L(a, \pi)$, and $q(x)=0$ for $x \in[0, a]$.

The following inverse problem is studied: given the spectra of the problems $L_{j}, j=0,1$, find the potential $q(x)$ and the coefficient $h$. Differential equations with delay arise in various problems of mathematics as well as in applications (see the monographs [22]-[25] and the references therein). Some results on the spectral theory of differential operators with delay can be found in $[10,18,24,26]$ and other works. The presence of delay in a mathematical model produces serious qualitative changes in the study of spectral problems. Therefore, up to now there are no comprehensive results in the inverse problem theory for operators with delay.

In the next section we study spectral properties of the boundary value problems (1)-(2), in particular, properties of the characteristic functions and the eigenvalues of $L_{j}$. In Section 3 we consider the inverse spectral problem of recovering the potential $q(x)$ and the coefficient $h$ from the given two spectra of the boundary value problems $L_{0}$ and $L_{1}$. We provide a uniqueness result for this inverse problem. More precisely, we prove that if the eigenvalues of $L_{j}(q, h)$ are the same as for the zero potential, then $q$ can be only zero.

\section{Characteristic functions and spectra}

Let $N \in \mathbb{N}$ be such that $a N<\pi \leq a(N+1)$, i.e. $a \in[\pi /(N+1), \pi / N)$. Let $C(x, \lambda), S(x, \lambda)$ and $\varphi(x, \lambda)$ be solutions of Eq. (1) under the initial conditions

$$
C(0, \lambda)=S^{\prime}(0, \lambda)=\varphi(0, \lambda)=1, \quad S(0, \lambda)=C^{\prime}(0, \lambda)=0, \quad \varphi^{\prime}(0, \lambda)=h
$$

For each fixed $x$, and $v=0,1$ the functions $C^{(v)}(x, \lambda), S^{(v)}(x, \lambda)$ and $\varphi^{(v)}(x, \lambda)$ are entire in $\lambda$ of order $1 / 2$, and

$$
\varphi(x, \lambda)=C(x, \lambda)+h S(x, \lambda) .
$$

Let $\lambda=\rho^{2}$ and $\rho=\sigma+i \tau$, i.e. $\sigma=\operatorname{Re} \rho, \tau=\operatorname{Im} \rho$.

\section{Lemma 1. The following representations hold}

$$
\varphi(x, \lambda)=\varphi_{0}(x, \lambda)+\varphi_{1}(x, \lambda)+\ldots+\varphi_{N}(x, \lambda), S(x, \lambda)=S_{0}(x, \lambda)+S_{1}(x, \lambda)+\ldots+S_{N}(x, \lambda),(4)
$$

where

$$
\left.\begin{array}{rl}
\varphi_{0}(x, \lambda)= & \cos \rho x+h \frac{\sin \rho x}{\rho}, S_{0}(x, \lambda)=\frac{\sin \rho x}{\rho}, \quad x \geq 0, \\
\varphi_{k}(x, \lambda)=\int_{k a}^{x} \frac{\sin \rho(x-t)}{\rho} q(t) \varphi_{k-1}(t-a, \lambda) d t, \\
S_{k}(x, \lambda)=\int_{k a}^{x} \frac{\sin \rho(x-t)}{\rho} q(t) S_{k-1}(t-a, \lambda) d t,
\end{array}\right\}
$$


for $x \geq k a$, and $\varphi_{k}(x, \lambda)=S_{k}(x, \lambda)=0$ for $x \leq k a$. Moreover, for $|\rho| \rightarrow \infty, v=0,1$, uniformly in $x$ the following estimates hold:

$$
\varphi_{k}^{(v)}(x, \lambda)=O\left(\rho^{v-k} \exp (|\tau|(x-k a))\right), \quad S_{k}^{(v)}(x, \lambda)=O\left(\rho^{v-k-1} \exp (|\tau|(x-k a))\right)
$$

Proof. The functions $\varphi(x, \lambda)$ and $S(x, \lambda)$ are the solutions of the integral equations

$$
\left.\begin{array}{c}
\varphi(x, \lambda)=\cos \rho x+h \frac{\sin \rho x}{\rho}+\int_{0}^{x} \frac{\sin \rho(x-t)}{\rho} q(t) \varphi(t-a, \lambda) d t, \\
S(x, \lambda)=\frac{\sin \rho x}{\rho}+\int_{0}^{x} \frac{\sin \rho(x-t)}{\rho} q(t) S(t-a, \lambda) d t .
\end{array}\right\}
$$

Solving integral equations (7) by the method of successive approximations we arrive at (4), where the functions $\varphi_{k}(x, \lambda)$ and $S_{k}(x, \lambda), k=\overline{1, N}$, are defined by (5). Moreover, it follows from (5) that

$$
\left.\begin{array}{l}
\varphi_{k}^{\prime}(x, \lambda)=\int_{k a}^{x} \cos \rho(x-t) q(t) \varphi_{k-1}(t-a, \lambda) d t \\
S_{k}^{\prime}(x, \lambda)=\int_{k a}^{x} \cos \rho(x-t) q(t) S_{k-1}(t-a, \lambda) d t
\end{array}\right\}
$$

for $x \geq k a$. Clearly, $\varphi_{0}^{(v)}(x, \lambda)=O\left(\rho^{v} \exp (|\tau| x)\right), S_{0}^{(v)}(x, \lambda)=O\left(\rho^{v-1} \exp (|\tau| x)\right)$. Using (5) and (8), we arrive at (6) by induction.

Corollary 1. The following representation holds

$$
\begin{aligned}
C(x, \lambda) & =C_{0}(x, \lambda)+C_{1}(x, \lambda)+\ldots+C_{N}(x, \lambda), \\
C_{0}(x, \lambda) & =\cos \rho x, \quad x \geq 0, \\
C_{k}(x, \lambda) & =\int_{k a}^{x} \frac{\sin \rho(x-t)}{\rho} q(t) C_{k-1}(t-a, \lambda) d t,
\end{aligned}
$$

for $x \geq k a$, and $C_{k}(x, \lambda)=0$ for $x \leq k a$. Moreover, for $|\rho| \rightarrow \infty, v=0,1$, uniformly in $x$ the following estimate holds:

$$
C_{k}^{(v)}(x, \lambda)=O\left(\rho^{v-k} \exp (|\tau|(x-k a))\right)
$$

Relations (9), (10) and (11) follow from (4), (5) and (6), respectively, with $h=0$.

Remark 1. The functions $C_{1}(x, \lambda), S_{1}(x, \lambda)$ and $\varphi_{1}(x, \lambda)$ depend linearly on the potential $q$. In 
particular, taking (5) and (10) into account, we calculate for $x \geq a$ :

$$
\left.\begin{array}{l}
C_{1}(x, \lambda)=\frac{\sin \rho(x-a)}{2 \rho} \int_{a}^{x} q(t) d t+\frac{1}{2 \rho} \int_{a}^{x} q(t) \sin \rho(x-2 t+a) d t, \\
C_{1}^{\prime}(x, \lambda)=\frac{\cos \rho(x-a)}{2} \int_{a}^{x} q(t) d t+\frac{1}{2} \int_{a}^{x} q(t) \cos \rho(x-2 t+a) d t, \\
S_{1}(x, \lambda)=-\frac{\cos \rho(x-a)}{2 \rho^{2}} \int_{a}^{x} q(t) d t+\frac{1}{2 \rho^{2}} \int_{a}^{x} q(t) \cos \rho(2 t-x-a) d t, \\
S_{1}^{\prime}(x, \lambda)=\frac{\sin \rho(x-a)}{2 \rho} \int_{a}^{x} q(t) d t+\frac{1}{2 \rho} \int_{a}^{x} q(t) \sin \rho(2 t-x-a) d t .
\end{array}\right\}
$$

Denote $\Delta_{j}(\lambda):=\varphi^{(j)}(\pi, \lambda), j=0,1$. The functions $\Delta_{j}(\lambda)$ are entire in $\lambda$ of order $1 / 2$. The zeros of $\Delta_{j}(\lambda)$ coincide with the eigenvalues of the boundary value problems $L_{j}(q, h)$ (counting with multiplicities). The function $\Delta_{j}(\lambda)$ is called the characteristic function for $L_{j}(q, h)$.

Lemma 2. For $|\rho| \rightarrow \infty$ the following asymptotical formulae are valid:

$$
\begin{aligned}
& \Delta_{0}(\lambda)=\cos \rho \pi+h \frac{\sin \rho \pi}{\rho}+\frac{\sin \rho(\pi-a)}{2 \rho} \int_{a}^{\pi} q(t) d t+o\left(\frac{1}{\rho} \exp (|\tau|(\pi-a))\right), \\
& \Delta_{1}(\lambda)=-\rho \sin \rho \pi+h \cos \rho \pi+\frac{\cos \rho(\pi-a)}{2} \int_{a}^{\pi} q(t) d t+o(\exp (|\tau|(\pi-a))) .
\end{aligned}
$$

Proof. Using (9), (11) and (12), we obtain

$$
\begin{aligned}
C(\pi, \lambda)= & \cos \rho \pi+\frac{\sin \rho(\pi-a)}{2 \rho} \int_{a}^{\pi} q(t) d t+\frac{1}{2 \rho} \int_{a}^{\pi} q(t) \sin \rho(\pi-2 t+a) d t \\
& +O\left(\rho^{-2} \exp (|\tau|(\pi-2 a))\right) .
\end{aligned}
$$

Since

$$
\int_{a}^{\pi} q(t) \sin \rho(\pi-2 t+a) d t=\frac{1}{2} \int_{-(\pi-a)}^{(\pi-a)} q((\pi+a-\xi) / 2) \sin \rho \xi d \xi=o(\exp (|\tau|(\pi-a))),
$$

it follows that

$$
C(\pi, \lambda)=\cos \rho \pi+\frac{\sin \rho(\pi-a)}{2 \rho} \int_{a}^{\pi} q(t) d t+o\left(|\rho|^{-1} \exp (|\tau|(\pi-a))\right) .
$$

Analogously we calculate

$$
\begin{aligned}
& C^{\prime}(\pi, \lambda)=-\rho \sin \rho \pi+\frac{\cos \rho(\pi-a)}{2} \int_{a}^{\pi} q(t) d t+o(\exp (|\tau|(\pi-a))), \\
& S(\pi, \lambda)=\frac{\sin \rho \pi}{\rho}-\frac{\cos \rho(\pi-a)}{2 \rho^{2}} \int_{a}^{\pi} q(t) d t+o\left(|\rho|^{-2} \exp (|\tau|(\pi-a))\right), \\
& S^{\prime}(\pi, \lambda)=\cos \rho \pi+\frac{\sin \rho(\pi-a)}{2 \rho} \int_{a}^{\pi} q(t) d t+o\left(|\rho|^{-1} \exp (|\tau|(\pi-a))\right) .
\end{aligned}
$$


By virtue of (3), one has

$$
\Delta_{j}(\lambda)=C^{(j)}(\pi, \lambda)+h S^{(j)}(\pi, \lambda), \quad j=0,1
$$

Substituting (15)-(16) into (17), we arrive at (13)-(14).

Lemma 3. The boundary value problem $L_{j}$ has a countable set of eigenvalues $\left\{\lambda_{n j}\right\}_{n \geq 0}, j=0,1$ (counting with multiplicities) and for $n \rightarrow \infty$ :

$$
\begin{aligned}
& \rho_{n 0}:=\sqrt{\lambda_{n 0}}=\left(n+\frac{1}{2}\right)+\frac{h}{\pi n}+\frac{\cos (n+1 / 2) a}{2 \pi n} \int_{a}^{\pi} q(t) d t+o\left(\frac{1}{n}\right), \\
& \rho_{n 1}:=\sqrt{\lambda_{n 1}}=n+\frac{h}{\pi n}+\frac{\cos n a}{2 \pi n} \int_{a}^{\pi} q(t) d t+o\left(\frac{1}{n}\right) .
\end{aligned}
$$

Proof. It follows from (14) that

$$
\Delta_{1}(\lambda)=-\rho \sin \rho \pi+g(\lambda), \quad|g(\lambda)| \leq C \exp (|\tau| \pi) .
$$

Here and below, the symbol "C" denotes various positive constants in estimates. Fix $\delta>0$. Denote $G_{\delta}:=\{\rho:|\rho-k| \geq \delta, k \in \mathbb{Z}\}$. Since $|\sin \rho \pi| \geq C \exp (|\tau| \pi)$ for $\rho \in G_{\delta}$, it follows from (20) that

$$
|\rho \sin \rho \pi|>|g(\lambda)|, \quad \rho \in G_{\delta}, \quad|\rho| \geq \rho^{*},
$$

for sufficiently large $\rho^{*}$. Let $\Gamma_{n}:=\left\{\lambda:|\lambda|=(n+1 / 2)^{2}\right\}$. Using (20), (21) and Rouché's theorem [27, p.125], we conclude that the number of zeros of $\Delta_{1}(\lambda)$ inside $\Gamma_{n}$ is equal to $n+1$. Thus, in the circle $|\lambda|<(n+1 / 2)^{2}$ there exist exactly $n+1$ eigenvalues of the boundary value problem $L_{1}: \lambda_{01}, \ldots, \lambda_{n 1}$. Applying now Rouche's theorem to the circle $\gamma_{n}(\delta):=\{\rho:|\rho-n| \leq \delta\}$, we conclude that for sufficiently large $n$, in $\gamma_{n}$ there is exactly one zero of $\Delta\left(\rho^{2}\right)$, namely $\rho_{n 1}=$ $\sqrt{\lambda_{n 1}}$. Since $\delta>0$ is arbitrary, it follows that

$$
\rho_{n 1}=n+\varepsilon_{n}, \quad \varepsilon_{n}=o(1), n \rightarrow \infty .
$$

Since $\Delta_{1}\left(\rho_{n 1}^{2}\right)=0$, it follows from (14) and (22) that

$$
\rho_{n 1} \sin \rho_{n 1} \pi=h \cos \rho_{n 1} \pi+\frac{\cos \rho_{n 1}(\pi-a)}{2} \int_{a}^{\pi} q(t) d t+o(1), \quad n \rightarrow \infty,
$$

and, consequently,

$$
n \sin \varepsilon_{n} \pi=h+\frac{\cos n a}{2} \int_{a}^{\pi} q(t) d t+o(1), \quad n \rightarrow \infty .
$$

This yields

$$
\varepsilon_{n}=\frac{h}{\pi n}+\frac{\cos n a}{2 \pi n} \int_{a}^{\pi} q(t) d t+o\left(\frac{1}{n}\right), \quad n \rightarrow \infty,
$$

and we arrive at (19). Relation (18) can be obtained by similar arguments. 
Lemma 4. The specification of the spectrum $\left\{\lambda_{n j}\right\}_{n \geq 0}$ uniquely determines the characteristic function $\Delta_{j}(\lambda)$ by the formulae

$$
\Delta_{0}(\lambda)=\prod_{n=0}^{\infty} \frac{\lambda_{n 0}-\lambda}{(n+1 / 2)^{2}}, \quad \Delta_{1}(\lambda)=\pi\left(\lambda_{01}-\lambda\right) \prod_{n=1}^{\infty} \frac{\lambda_{n 1}-\lambda}{n^{2}} .
$$

Proof. By Hadamard's factorization theorem [27, p.289], $\Delta_{1}(\lambda)$ is uniquely determined up to a multiplicative constant by its zeros:

$$
\Delta_{1}(\lambda)=C \prod_{n=0}^{\infty}\left(1-\frac{\lambda}{\lambda_{n 1}}\right)
$$

(the case when $\Delta_{1}(0)=0$ requires minor modifications). Consider the function

$$
\tilde{\Delta}_{1}(\lambda)=-\rho \sin \rho \pi=-\lambda \pi \prod_{n=1}^{\infty}\left(1-\frac{\lambda}{n^{2}}\right) .
$$

Then

$$
\frac{\Delta_{1}(\lambda)}{\tilde{\Delta}_{1}(\lambda)}=\frac{C\left(\lambda-\lambda_{01}\right)}{\lambda_{01} \pi \lambda} \prod_{n=1}^{\infty} \frac{n^{2}}{\lambda_{n 1}} \prod_{n=1}^{\infty}\left(1+\frac{\lambda_{n 1}-n^{2}}{n^{2}-\lambda}\right) .
$$

Taking (14) and (19) into account we calculate

$$
\lim _{\lambda \rightarrow-\infty} \frac{\Delta_{1}(\lambda)}{\tilde{\Delta}_{1}(\lambda)}=1, \quad \lim _{\lambda \rightarrow-\infty} \prod_{n=1}^{\infty}\left(1+\frac{\lambda_{n 1}-n^{2}}{n^{2}-\lambda}\right)=1,
$$

and hence

$$
C=\pi \lambda_{01} \prod_{n=1}^{\infty} \frac{\lambda_{n 1}}{n^{2}}
$$

Substituting this into (24) we arrive at (23) for $\Delta_{1}(\lambda)$. For the function $\Delta_{0}(\lambda)$, the arguments are similar.

Denote

$$
L(\rho):=\Delta_{1}(\lambda)+i \rho \Delta_{0}(\lambda)
$$

The function $L(\rho)$ is entire in $\rho$ of exponential type, and $L(\rho)$ is the characteristic function for the Redge-type boundary value problem for Eq. (1) with the boundary conditions $y^{\prime}(0)-$ $h y(0)=y^{\prime}(\pi)+i \rho y(\pi)=0$. Clearly,

$$
L(\rho)=L_{0}(\rho)+L_{1}(\rho)+\ldots+L_{N}(\rho), \quad L_{k}(\rho)=\varphi_{k}^{\prime}(\pi, \rho)+i \rho \varphi_{k}(\pi, \rho) .
$$

In particular,

$$
L_{0}(\rho)=(-\rho \sin \rho \pi+h \cos \rho \pi)+(i \rho \cos \rho \pi+i h \sin \rho \pi),
$$

and consequently,

$$
L_{0}(\rho)=(i \rho+h) \exp (i \rho \pi)
$$


Let us calculate $L_{1}(\rho)$. For this purpose we use (5), (10), (12) and (26):

$$
\begin{aligned}
L_{1}(\rho)= & \left(C_{1}^{\prime}(\pi, \lambda)+h S_{1}^{\prime}(\pi, \lambda)\right)+i \rho\left(C_{1}(\pi, \lambda)+h S_{1}(\pi, \lambda)\right) \\
= & \frac{\cos \rho(\pi-a)}{2} \int_{a}^{\pi} q(t) d t+\frac{1}{2} \int_{a}^{\pi} q(t) \cos \rho(\pi-2 t+a) d t \\
& +\frac{h \sin \rho(\pi-a)}{2 \rho} \int_{a}^{\pi} q(t) d t+\frac{h}{2 \rho} \int_{a}^{\pi} q(t) \sin \rho(2 t-\pi-a) d t \\
& +\frac{i \sin \rho(\pi-a)}{2} \int_{a}^{\pi} q(t) d t+\frac{i}{2} \int_{a}^{\pi} q(t) \sin \rho(\pi-2 t+a) d t \\
& -\frac{i h \cos \rho(\pi-a)}{2 \rho} \int_{a}^{\pi} q(t) d t+\frac{i h}{2 \rho} \int_{a}^{\pi} q(t) \cos \rho(2 t-\pi-a) d t .
\end{aligned}
$$

Therefore,

$$
\begin{aligned}
L_{1}(\rho)= & \left(\frac{1}{2}+\frac{h}{2 i \rho}\right) \exp (i \rho(\pi-a)) \int_{a}^{\pi} q(t) d t \\
& +\left(\frac{1}{2}-\frac{h}{2 i \rho}\right) \exp (i \rho(\pi+a)) \int_{a}^{\pi} q(t) \exp (-2 i \rho t) d t .
\end{aligned}
$$

Lemma 5. For $\tau \geq 0,|\rho| \rightarrow \infty, k \geq 1$, the following estimate holds

$$
L_{k}(\rho)=O\left(\frac{1}{\rho^{k-1}} \int_{k a}^{\pi}|q(t) \exp (-i \rho(2 t-\pi-k a))| d t\right) .
$$

Proof. Using (26), (5) and (8), we calculate

$$
L_{k}(\rho)=\int_{k a}^{\pi}(\cos \rho(\pi-t)+i \sin \rho(\pi-t)) q(t) \varphi_{k-1}(t-a, \lambda) d t,
$$

and consequently,

$$
L_{k}(\rho)=\int_{k a}^{\pi} \exp (i \rho(\pi-t)) q(t) \varphi_{k-1}(t-a, \lambda) d t, \quad k \geq 1 .
$$

On the other hand, it follows from (6) that

$$
\left|\varphi_{k-1}(t-a, \lambda)\right| \leq C|\rho|^{1-k}|\exp (-i \rho(t-k a))|, \tau \geq 0 .
$$

Substituting (30) into (29), we arrive at (28).

\section{The inverse problem}

In this section we consider the following inverse problem: given two spectra $\left\{\lambda_{n j}\right\}_{n \geq 0}$, $j=0,1$, find $q(x)$ and $h$. In order to formulate a uniqueness result for this inverse problem, we consider together with $L_{j}$ the boundary value problems $\tilde{L}_{j}:=L_{j}(\tilde{q}, \tilde{h})$ of the same form but with different $\tilde{q}$ and $\tilde{h}$. We agree that if a certain symbol $\beta$ denotes an object related to $L_{j}$, then $\tilde{\beta}$ will denote the analogous object related to $\tilde{L}_{j}$. 
Let $\left\{\tilde{\lambda}_{n j}\right\}_{n \geq 0}, j=0,1$, be the eigenvalues of the boundary value problems $\tilde{L}_{j}$ with $\tilde{q}(x) \equiv 0$. Let $\tilde{\Delta}_{j}(\lambda)$ be the characteristic functions of $\tilde{L}_{j}$, and

$$
\tilde{L}(\rho)=\tilde{\Delta}_{1}(\lambda)+i \rho \tilde{\Delta}_{0}(\lambda)
$$

Theorem 1. If $\lambda_{n j}=\tilde{\lambda}_{n j}$ for all $n \geq 0, j=0,1$, then $q(x)=\tilde{q}(x)$ a.e. on $(a, \pi)$ and $h=\tilde{h}$.

Proof. 1) By virtue of Lemma 4, one has

$$
\Delta_{0}(\lambda)=\tilde{\Delta}_{0}(\lambda), \quad \Delta_{1}(\lambda)=\tilde{\Delta}_{1}(\lambda)
$$

Using (25) and (31) we get

$$
L(\rho)=\tilde{L}(\rho)
$$

Since $\tilde{q}(x) \equiv 0$, it follows from (18)-(19) and (29) that

$$
\int_{a}^{\pi} q(t) d t=0, \quad h=\tilde{h}, \quad \tilde{L}(\rho) \equiv L_{0}(\rho)=(i \rho+h) \exp (i \rho \pi) .
$$

In particular, (33) and (27) yield

$$
L_{1}(\rho)=\left(\frac{1}{2}-\frac{h}{2 i \rho}\right) \exp (i \rho(\pi+a)) \int_{a}^{\pi} q(t) \exp (-2 i \rho t) d t .
$$

Denote $L^{+}(\rho):=L_{2}(\rho)+\ldots+L_{N}(\rho)$ for $N \geq 2$, and $L^{+}(\rho) \equiv 0$ for $N=1$. Using (26), (32) and (33), we obtain

$$
L_{1}(\rho) \equiv-L^{+}(\rho)
$$

2) First of all, we note that if $q(x)=0$ a.e. on $(2 a, \pi)$, then $q(x)=0$ a.e. on $(a, \pi)$. Indeed, under this assumption we have $L^{+}(\rho) \equiv 0$, and according to (35) we infer $L_{1}(\rho) \equiv 0$. Using (34) we obtain

$$
\int_{a}^{\pi} q(t) \exp (-2 i \rho t) d t \equiv 0
$$

and consequently, $q(x)=0$ a.e. on $(a, \pi)$. In particular, this finishes the proof for $N=1$, since in this case we have $2 a \geq \pi$ and automatically $L^{+}(\rho) \equiv 0$.

3) Let $N \geq 2$. Fix $v=\overline{0,2 N-3}$. Let us prove that

$$
\text { if } q(x)=0 \text { a.e. on }\left(\pi-\frac{v a}{2}, \pi\right) \text {, then } q(x)=0 \text { a.e. on }\left(\pi-\frac{(v+1) a}{2}, \pi\right) \text {. }
$$

Indeed, it follows from (28) that

$$
L_{2}(\rho)=O\left(\frac{1}{\rho} \int_{2 a}^{\pi-v a / 2}|q(t) \exp (-i \rho(2 t-\pi-2 a))| d t\right), \quad \tau \geq 0,|\rho| \rightarrow \infty .
$$


Let $\pi-v a / 2>2 a$, otherwise we arrive at the situation in 2) and the proof is finished. Then in the integral we have $2 a-\pi \leq 2 t-\pi-2 a \leq \pi-(v+2) a$, which yields

$$
L_{2}(\rho)=O\left(\frac{1}{\rho} \exp (-i \rho(\pi-(v+2) a))\right), \quad \tau \geq 0,|\rho| \rightarrow \infty .
$$

For $k>2$, the functions $L_{k}(\rho)$ have less growth than in (37). This means that

$$
L^{+}(\rho)=O\left(\frac{1}{|\rho|} \exp (-i \rho(\pi-(v+2) a))\right), \quad \tau \geq 0,|\rho| \rightarrow \infty .
$$

It follows from (34), (35) and (38) that

$$
\exp (i \rho(\pi+a)) \int_{a}^{\pi-v a / 2} q(t) \exp (-2 i \rho t) d t=O\left(\frac{1}{\rho} \exp (-i \rho(\pi-(v+2) a))\right), \tau \geq 0,|\rho| \rightarrow \infty,
$$

or, which is the same,

$$
\exp (i \rho(2 \pi-(v+1) a)) \int_{a}^{\pi-v a / 2} q(t) \exp (-2 i \rho t) d t=O\left(\frac{1}{\rho}\right), \quad \tau \geq 0,|\rho| \rightarrow \infty .
$$

Moreover, one has

$$
\int_{a}^{\pi-(v+1) a / 2} q(t) \exp (-2 i \rho t) d t=O(\exp (-i \rho(2 \pi-(v+1) a)), \quad \tau \geq 0,|\rho| \rightarrow \infty .
$$

Let us introduce the function

$$
F(\rho):=\exp (i \rho(2 \pi-(v+1) a)) \int_{\pi-(v+1) a / 2}^{\pi-v a / 2} q(t) \exp (-2 i \rho t) d t .
$$

The function $F(\rho)$ is entire in $\rho$. Clearly, $F(\rho)=O(1)$ for $\tau \leq 0$. On the other hand, it follows from (39) and (40) that $F(\rho)=O(1)$ for $\tau \geq 0$. By Liouville's theorem [27, p.77], $F(\rho) \equiv C$-const. Since $F(\rho)=o(1)$ for real $\rho,|\rho| \rightarrow \infty$, it follows that $F(\rho) \equiv 0$, i.e.

$$
\int_{\pi-(v+1) a / 2}^{\pi-v a / 2} q(t) \exp (-2 i \rho t) d t \equiv 0 .
$$

This yields $q(x)=0$ a.e. on the interval $(\pi-(v+1) a / 2, \pi-v a / 2)$, i.e. (36) is proved.

Applying proposition (36) successively for $v=0,1, \ldots, 2 N-3$, we obtain $q(x)=0$ a.e. on the interval $(\pi-(N-1) a, \pi) \supset(2 a, \pi)$. According to 2$)$ we get $q(x)=0$ a.e. on $(a, \pi)$. Theorem 1 is proved.

\section{Acknowledgement}

This work was supported in part by Grants 1.1436.2014K, 1.1660.2017/PCh of the Ministry of Education and Science of RF and by Grants 15-01-04864, 16-01-00015 of Russian Foundation for Basic Research. 


\section{References}

[1] V. A. Marchenko, Sturm-Liouville Operators and Their Applications. Naukova Dumka, Kiev, 1977; English transl., Birkhäuser, 1986.

[2] B. M. Levitan, Inverse Sturm-Liouville Problems, Nauka, Moscow, 1984 (Russian); English transl., VNU Sci.Press, Utrecht, 1987.

[3] G. Freiling and V. A. Yurko, Inverse Sturm-Liouville Problems and Their Applications. NOVA Science Publishers, New York, 2001.

[4] V. A. Yurko, Introduction to the Theory of Inverse Spectral Problems, Moscow, Fizmatlit, 2007.

[5] V. A. Yurko, Method of Spectral Mappings in the Inverse Problem Theory, Inverse and Ill-posed Problems Series. VSP, Utrecht, 2002.

[6] V. A. Yurko, Inverse Spectral Problems for Differential Operators and Their Applications, Gordon and Breach Science Publishers, Amsterdam, 2000.

[7] R. Beals, P. Deift and C. Tomei, Direct and Inverse Scattering on the Line, Mathematica Surveys and Monographs, 28. AMS, Providence, RI, 1988.

[8] V. A. Yurko, An inverse problem for integral operators, Mat. Zametki, 37 (1985), no.5, 690-701; English transl. in Math. Notes 37 (1985) no 5-6, 378-385.

[9] M. S. Eremin, An inverse problem for a second-order integro-differential equation with a singularity, Diff. Uravn., 24 (1988), no.2, 350-351.

[10] M. Pikula, Determination of a Sturm-Liouville-type differential operator with delay argument from two spectra, Mat. Vesnik, 43 (1991), no.3-4, 159-171 (Russian).

[11] V. A. Yurko, An inverse problem for integro-differential operators, Matem. Zametki, 50 (1991), no.5, 134-146 (Russian); English transl. in Math. Notes, 50 (1991), no.5-6, 1188-1197.

[12] K. V. Kravchenko, On differential operators with nonlocal boundary conditions, Diff. Uravn., 36 (2000), no. 4, 464-469; English transl. in Diff. Eqns., 36 (2000), no.4, 517-523.

[13] S. A. Buterin, The inverse problem of recovering the Volterra convolution operator from the incomplete spectrum of its rank-one perturbation, Inverse Problems, 22 (2006), 2223-2236.

[14] S. A. Buterin, Inverse spectral reconstruction problem for the convolution operator perturbed by a onedimensional operator, Matem. Zametki, 80 (2006), no.5, 668-682 (Russian); English transl. in Math. Notes, 80 (2006), no.5, 631-644.

[15] S. A. Buterin, On an inverse spectral problem for a convolution integro-differential operator, Results in Math., 50 (2007), no.3-4, 173-181.

[16] Yu. V. Kuryshova, The inverse spectral problem for integro-differential operators, Matem. Zametki, 81 (2007), no. 6, 855-866 (Russian); English translation in Math. Notes, 81 (2007), no. 5-6, 767-777.

[17] S. A. Buterin, On the reconstruction of a convolution perturbation of the Sturm-Liouville operator from the spectrum, Diff. Uravn., 46 (2010), 146-149 (Russian); English transl. in Diff. Eqns., 46 (2010), 150-154.

[18] G. Freiling and V. A. Yurko, Inverse problems for Sturm-Liouville differential operators with a constant delay, Appl. Math. Lett., 25 (2012), 1999-2004.

[19] V. A. Yurko, An inverse spectral problems for integro-differential operators, Far East J. Math. Sci., 92 (2014), no.2, 247-261.

[20] S. A. Buterin and A. E. Choque Rivero, On inverse problem for a convolution integro-differential operator with Robin boundary conditions, Appl. Math. Lett., 48 (2015), 150-155.

[21] N. P. Bondarenko and S. A. Buterin, On recovering the Dirac operator with an integral delay from the spectrum, Res. Math. (2016), DOI 10.1007/s00025-016-0568-1.

[22] A. D. Myshkis, Linear Differential Equations with a Delay Argument, Moscow, Nauka, 1951.

[23] R. Bellman and K. L. Cooke, Differential-Difference Equations, The RAND Corp. R-374-PR, 1963.

[24] S. B. Norkin, Second Order Differential Equations with a Delay Argument, Moscow, Nauka, 1965.

[25] J. Hale, Theory of Functional-Differential Equations, Springer-Verlag, New York, 1977.

[26] V. V. Vlasov, On the solvability and properties of solutions of functional-differential equations in a Hilbert space, Mat. Sb., 186 (1995), no.8, 67-92 (Russian); English transl. in Sb. Math., 186 (1995), no. 8, 1147-1172. 
[27] J. B. Conway, Functions of One Complex Variable, 2nd ed., vol.I, Springer-Verlag, New York, 1995.

Department of Mathematics, Saratov University, Astrakhanskaya 83, Saratov 410012, Russia.

E-mail: ButerinSA@info.sgu.ru

Department of Mathematics, Saratov University, Astrakhanskaya 83, Saratov 410012, Russia.

E-mail: YurkoVA@info.sgu.ru

Department of Mathematics, Informatics and Physics, University East Sarajevo, Alekse Šantica 1, East Sarajevo, Bosnia and Herzegovina

E-mail: pikulam1947@gmail.com 